\title{
MARPE Guide: A Case Report
}

\author{
Bruno L Minervino ${ }^{1}$, Maurício Barriviera² ${ }^{2}$ Marcelo de Morais Curado ${ }^{3}$, Luiz G Gandini ${ }^{4}$
}

\begin{abstract}
Aim: This paper presents a prototyped surgical guide for placement of MARPE-mini-implant-assisted rapid palatal expander.

Background: Investigations conducted in the last decades have increased the technological resources used in orthodontics. This scientific advance applies to several clinical procedures, including the planning of devices used in guided surgeries, using computed tomography images and intraoral scanning.

Case description: This case report presents maxillary expansion in an adult patient (18 years and 5 months), using the MARPE technique, by virtual planning and fabrication of a surgical guide (laser-printed on a 3D printer MoonRay D225-SprintRay, 2014), after merging of 3 files: digital file of the expander (.stl), computed tomography of the suture, and intraoral scanning of the patient, using the planning software NemoStudio (version 20122, 16.50.0.56).

Conclusion: Fabrication of a surgical guide for the MARPE technique provides an accurate transfer of the virtual planning to the surgical procedure. It allows three-dimensional orientation of the expander position and perforations of mini-implants, which are necessary to establish anchorage in areas with sufficient bone, assuring the system stability and a successful procedure.

Clinical significance: The mini-implant assisted rapid palatal expansion (MARPE) has been investigated as a promising option for correction of malocclusion related with maxillary atresia in adult patients and is an option to orthognathic surgery. Digital treatment planning is fundamental to establish individual, reproducible, and accurate parameters, as in the present case, which evidenced significant benefits in both occlusal and respiratory aspects.
\end{abstract}

Keywords: MARPE, Midpalatal suture, Rapid palatal expansion, Surgical guide.

The Journal of Contemporary Dental Practice (2019): 10.5005/jp-journals-10024-2649

\section{BACKGROUND}

Technological advances and detailed imaging studies have allowed the utilization of virtual planning. The digital workflow in orthodontics consists of the triad digital models, orthodontic software, and 3D printer, and allows faster digital planning of cases, enhancing the communication between professionals and patients without the need to store physical documents. Also, the accessibility and easy utilization of this technology allows its broad utilization for diagnosis and treatment planning., ${ }^{1,2}$

The virtual models in Standard Triangle Language (STL) derivated from the intra-oral scanners, associated with cone beam computed tomography (CBCT) and a software for virtual planning are fundamental for 3D orthodontic preparation. ${ }^{2}$

Virtual planning in orthodontics is used for fabrication of guides for indirect bonding, virtual positioning of brackets, fabrication of aligners, setups, surgical simulations, and for fabrication of guides for orthodontic mini-implants, bone implants, or orthognathic surgery. ${ }^{3-6}$ However, utilization of this protocol is still limited in the planning of posterior transverse malocclusions.

The prevalence of maxillary transverse deficiency ranges from $8 \%$ to $23 \%$ in mixed and deciduous dentitions and less than $10 \%$ in adults and it may worsen over time, impairing the facial growth and development. ${ }^{7}$ In growing patients, the elective treatment of maxillary atresia is performed by rapid maxillary expansion (RME). However, in adult patients, the technique indicated is the surgically assisted rapid palatal expansion (SARPE). ${ }^{8}$

The literature reports that Hyrax and Haas expanders are most often used for RME. However, these devices present some side effects because they do not provide a purely skeletal expansion, also promoting tooth inclinations and rotation of maxillary segments, relapse, excessive compression of the periodontium, gingival
${ }^{1,4}$ Department of Orthodontics, School of Dentistry at Araraquara, UNESP São Paulo State University, Araraquara, São Paulo, Brazil

${ }^{2}$ Department of Oral Radiology, School of Dentistry, Catholic University of Brasilia, Brasilia, Distrito Federal, Brazil

${ }^{3}$ Department of Orthodontics, Planalto University Center, School of Dentistry, Brasilia, Distrito Federal, Brazil

Corresponding Author: Bruno L Minervino, Department of Orthodontics, School of Dentistry at Araraquara, UNESP São Paulo State University, Araraquara, São Paulo, Brazil, Phone: +55 6198185 0378, e-mail: dr.brunominervino@gmail.com

How to cite this article: Minervino BL, Barriviera M, et al. MARPE Guide: A Case Report. J Contemp Dent Pract 2019;20(9):1102-1107.

Source of support:This study was financed in part by the Coordenação de Aperfeiçoamento de Pessoal de Nível Superior-Brasil (CAPES) Finance Code 001

Conflict of interest: None

recession, fenestrations of the buccal bone plate, necrosis of palatal tissue, pain, and edema. ${ }^{9-11}$

More recent studies described that it is possible to perform palatal expansion without surgery, even in individuals after the growth period, by a technique known as mini-implant assisted rapid palatal expander (MARPE), initially developed by Won Monn at the University of California at Los Angeles (UCLA). The main difference is that MARPE applies forces directly on the maxillary bone segments and midpalatal suture, while in RME the forces act on the pterygomaxillary, nasomaxillary, and zygomaxillary regions. Therefore, the MARPE device is an option to the SARPE technique. ${ }^{10,12,13}$

() The Author(s). 2019 Open Access This article is distributed under the terms of the Creative Commons Attribution 4.0 International License (https://creativecommons. org/licenses/by-nc/4.0/), which permits unrestricted use, distribution, and non-commercial reproduction in any medium, provided you give appropriate credit to the original author(s) and the source, provide a link to the Creative Commons license, and indicate if changes were made. The Creative Commons Public Domain Dedication waiver (http://creativecommons.org/publicdomain/zero/1.0/) applies to the data made available in this article, unless otherwise stated. 
Aiming to assure a predictable and less complex surgical step, this study presents a 3D surgical guide for placement of MARPE expander and mini-implants.

\section{Case Description}

Patient EPC, aged 18 years and 5 months, with the chief complaint of posterior skeletal bilateral crossbite, was presented for orthodontic treatment at the Specialization Course of the Brazilian Orthodontics Association-ABO at Brasília (Taguatinga, Brazil). The patient had not been previously referred for orthodontic treatment. After considerations about the objectives, limitations, and possible side effects, the proposed treatment was accepted.

The MARPE surgical guide and adequacy of position of expander and mini-implants were performed on the software NemoStudio (version 20122, 16.50.0.56), a Spanish planning software developed for utilization of bone implants, which was adapted to fabricate the MARPE guide.

For digital planning, it was necessary to obtain and merge the digital files of the expander (.stl), computed tomography of the midpalatal suture, and intraoral scanning of the patient. The scanned digital model of the patient was obtained using an intraoral scanner (CS3600 ${ }^{\circledR}$, Carestream, Rochester, NY, USA). The .stl file of the MARPE expander was provided by the company PecLab (Belo Horizonte, Brazil).

The DICOM file of cone beam computed tomography, with lip soft tissue retraction, was obtained using a tomography machine Prexion with $0.1 \mathrm{~mm}$ voxel, $0.5 \mathrm{~mm}$ focal point, and 360-degree rotation. ${ }^{14}$ Therefore, the coronal and axial tomographic sections allowed a more detailed evaluation of the degree of bone maturation of the midpalatal suture and its interdigitation. ${ }^{15}$

Following, intraoral scanning of the maxilla was superimposed to the computed tomography, using points at the teeth region as reference to allow determination of the correct position between intraoral scanning and the tomography. After this initial merging, the third digital file, namely the stereography of the MARPE expander, was also merged. After merging of the three files (tomography, scanning, and stl file of the expander), the expander and four mini-implants were positioned using the software NemoStudio (Fig. 1).

Concerning the expander position, the first step is to select the most adequate size to the patient's palate, since it is available in different sizes $(6 \mathrm{~mm}, 9 \mathrm{~mm}$, or $11 \mathrm{~mm})$. Treatment planning is important to avoid tissue compression around the expander, requiring analysis throughout the appliance, adapted to the palatal curvature. $^{8,16}$

For selection of mini-implants, the thickness of bone and palatal mucosa are individually calculated to determine the length of screw and transmucosal collar of each device, according to each site of placement no. 1, 2, 3, and 4. In positions I, II, and III, $15 \mathrm{~mm}$ mini-implants were used $(22 \mathrm{~mm}$ screw and $8 \mathrm{~mm}$ transmucosal collar), and a $9 \mathrm{~mm}$ mini-implant was used in position IV owing to bone limitation ( $5 \mathrm{~mm}$ screw and $4 \mathrm{~mm}$ transmucosal collar), using mini-implants of the company PecLab (Belo Horizonte, Brazil, Fig. 1). This stage aimed to assure anchorage to allow primary stability of mini-implants and a better relationship of the transmucosal collar with the surrounding tissue and also the expander. ${ }^{17,18}$

After determining the MARPE position on the digital model of intraoral scanning, the stereography files (.stl) were exported from NemoStudio to the software Autodesk Meshmixer (Autodesk, Inc. 20122-version 3.2.322), in which the merging tools were used between the.stl files to stitch the two files into one.

After this stage, this single file was imported back to NemoStudio. This allows achievement of a guide with a laser 3D printer (SprintRay, 2014), using an autoclavable resin (Surgical Guide-MoonRay) (Fig. 2).

After surgery, it was possible to observe maxillary expansion in the patient outside the growth period (18 years and 5 months), with activation of $2 / 4$ turns per day, for 12 days $(6 \mathrm{~mm})$, without the need of bands (Fig. 3).

Finally, cone-beam computed tomography (CBCT) images were obtained before (Fig. 4) and after expansion (Fig. 5), which clearly show opening of the midpalatal suture throughout its extent.

\section{Discussion}

The new technologies available in orthodontics are fundamental to add resources for diagnosis and planning, since they provide real benefits to the patient. The manipulation of three-dimensional images allows virtual planning using CAD (computer-aided design) software, as well as customization of orthodontic devices by CAM (computer-aided manufacturing), such as for fabrication of surgical guides, which were initially used in implantology and endodontics. ${ }^{19,20}$

The surgical guide is an important tool for correct positioning of fixtures, aiming to achieve a perfect interrelation of digital

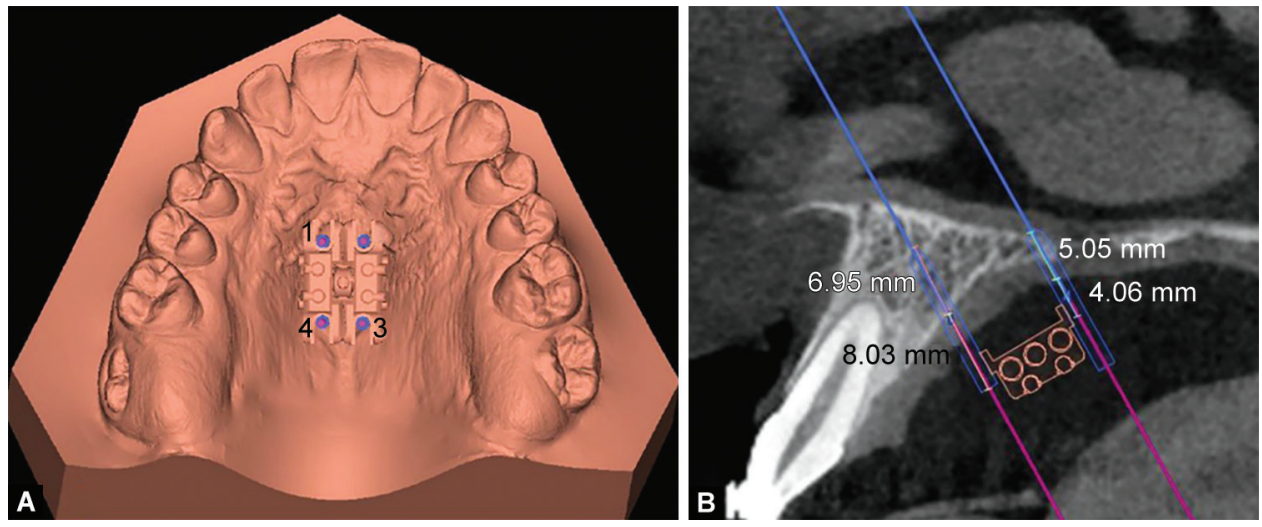

Figs $1 \mathrm{~A}$ and B: Digital model presenting planning of positioning of the MARPE expander and mini-implants HS—PecLab (Belo Horizonte, BRAZIL). (A) Expander positioning at $6 \mathrm{~mm}$ in mesiodistal and axial directions, without compression of surrounding tissues; (B) Planning of $15 \mathrm{~mm}$ miniimplants in position no. II (anterior region, $6.95 \mathrm{~mm}$ of bone and 8.03 of transmucosal collar), and $9 \mathrm{~mm}$ in position no. IV (posterior region, 5.05 $\mathrm{mm}$ of bone and $4.06 \mathrm{~mm}$ of transmucosal collar), due to the little bone quantity 

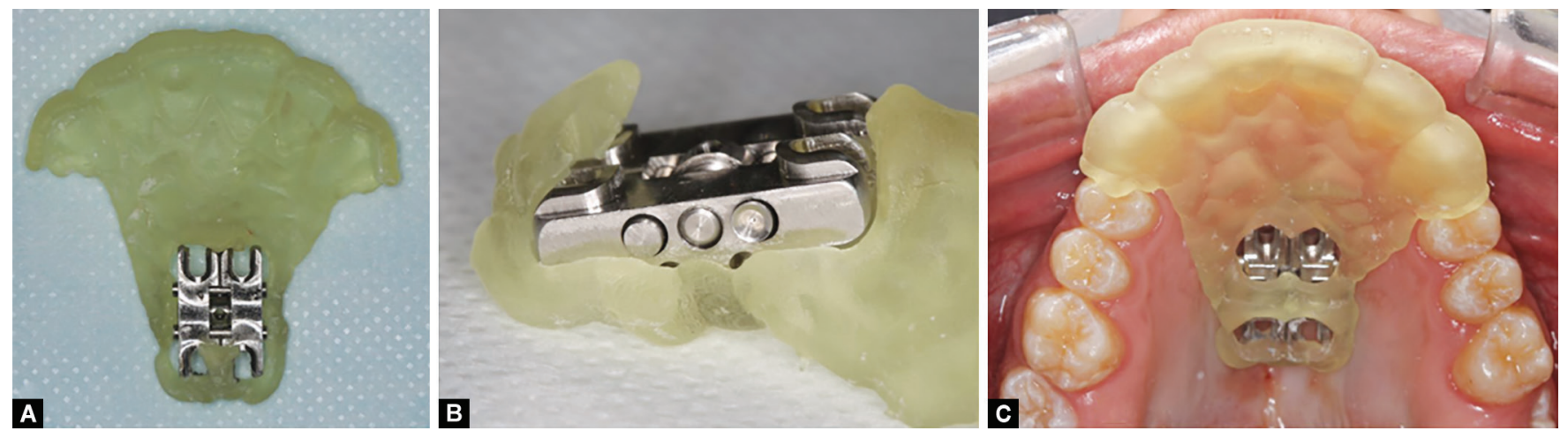

Figs 2A to C: Surgical guide printed in 3D using autoclavable resin (MoonRay). (A and B) The guide adapted to the orthodontic expander; (C) Occlusal photograph of the device at the moment of surgery and without utilization of orthodontic bands, because the rods were removed from the body of the original expander
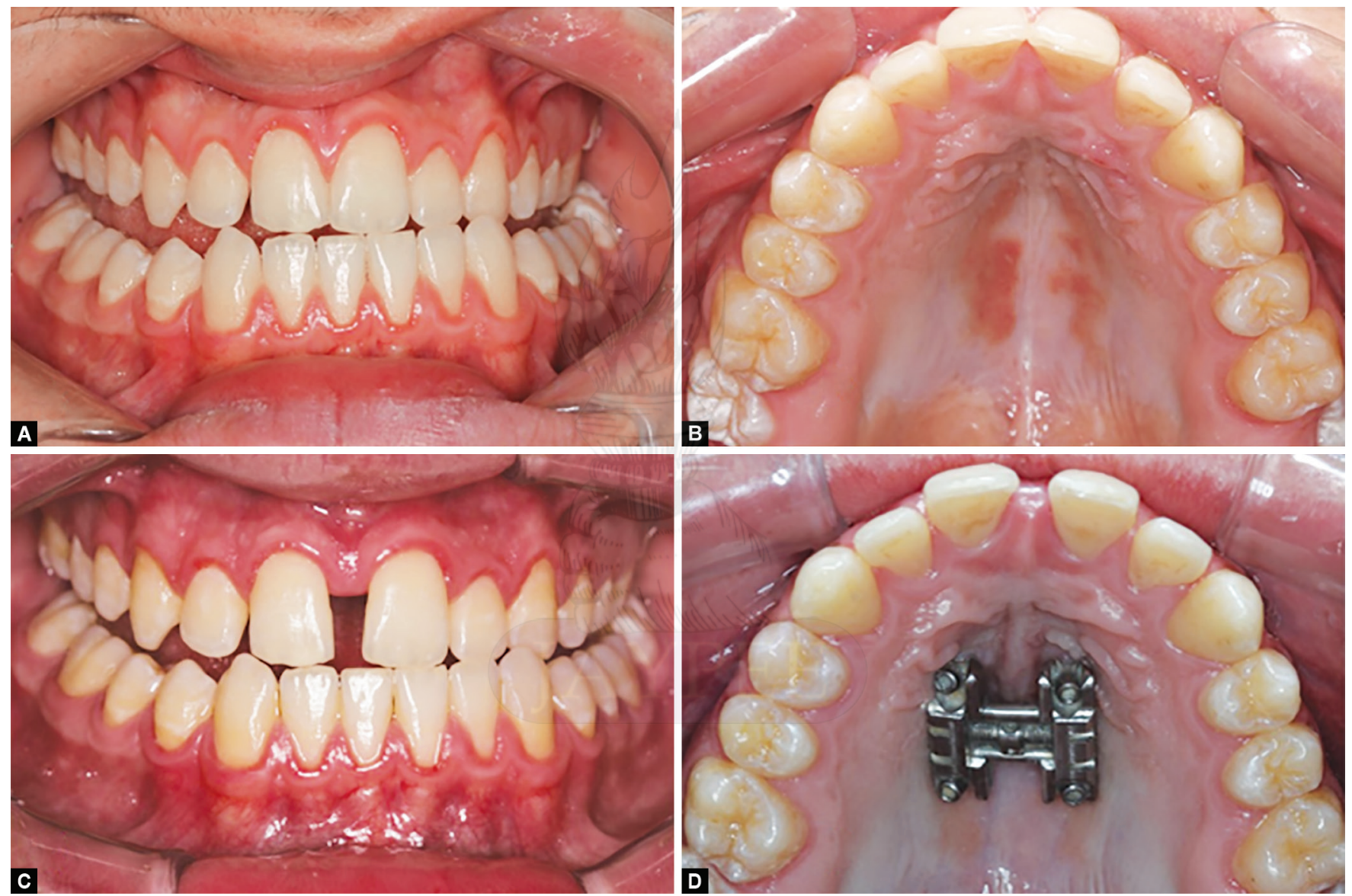

Figs 3A to D: Successful maxillary expansion. (A and B) Initial frontal and occlusal photographs; (C and D) Frontal and occlusal photographs after expansion; (C) Presence of interincisal diastema without inclination of posterior teeth

planning for actual placement. For this guide to adequately perform its functions, it should be adapted to the surgical site without impairing the surgeon's observation and access. This reverse planning involves stages previous to placement of specific devices.

Concerning orthodontics, all records may be digitally obtained and sent to the laboratories for fabrication of appliances, guides for indirect bonding or aligners, allowing the orthodontists to initiate the treatment considering the final objective, thus simplifying the mechanics toward a pre-established outcome. ${ }^{1,2,4}$ The placement of brackets in a single session or the fabrication of a series of aligners allows faster and customized treatments. ${ }^{21}$

The prognosis of RME in adult individuals is extremely doubtful and contraindicates utilization of the technique (RME) owing to the increased interdigitation of maxillary sutures and stiffness of adjacent structures. ${ }^{9,11}$ Therefore, it is important to combine the knowledge of basic sciences and the evolution of new technologies, aiming to establish safer therapeutic approaches. ${ }^{18}$

By direct force application on the palatal bone, the rapid palatal expansion anchored on orthodontic mini-implants 

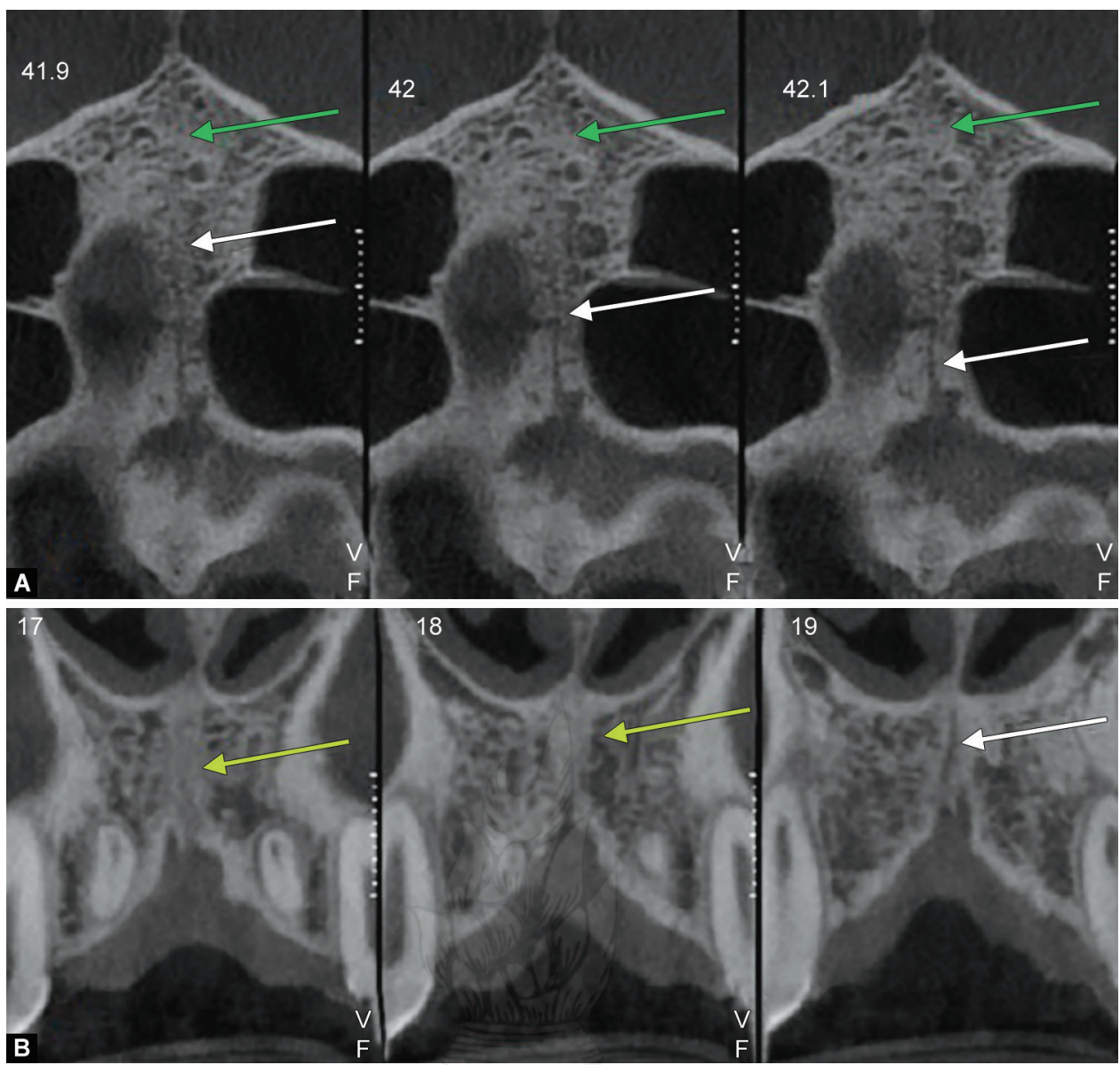

Figs 4A and B: Initial CBCT based on axial (A) and coronal (B) images. (A) Sections 41.9, 42.0, and 42.1: observe the presence of hypodense images compatible with non-calcified intermaxillary suture at the posterior middle region (white arrows), while the anterior region exhibits isolated calcification areas (green arrows); (B) Sections 17.0 and 18.0 and 19.0: observe the presence of slightly calcified intermaxillary suture (green arrows), and non-calcified at the inferior and superior regions (white arrow)

(MARPE-mini-implant assisted rapid palatal expansion) presents as an effective option for the treatment of maxillary atresia and crossbite, especially in adult patients. This allows achievement of purely skeletal expansion, even in cases with some degree of palatal suture closure. ${ }^{18,22}$

The MARPE technique is the option of choice in relation to the SARPE technique, ${ }^{12}$ providing more reliable results compared to the traditional procedure (REM). It presents several benefits and advantages: greater vertical control in dolichofacial patients, minimum tooth inclination, lower risk of periodontal side effects as gingival retraction and root resorption, increased maxillary protrusion when associated with facial mask therapy, greater stability, reduced relapse, direct action of forces on the midpalatal suture, and enhanced nasal flow. ${ }^{12,13,23,24}$

Initially, there are two fundamental aspects concerning planning for placement of Marpe: suture evaluation by CBCT to assess the possibility of expansion, ${ }^{15,25}$ and three-dimensional positioning of both expander and mini-implants to assure insertion in an area with bone support, since the broad anatomical variability of the palate and soft tissues, in regions with reduced or absent bone thickness, contraindicates its placement leading to procedure failure. ${ }^{18}$

The technique of mini-implant-assisted rapid palatal expansion became safer by assessment of the midpalatal suture on cone beam tomography machines. Using an iCAT machine, it is possible to score the stage of suture maturation (A-E), using axial sections, ${ }^{25}$ or on a tomography machine with better image resolution (Prexion), using axial and coronal sections. ${ }^{15}$

Concerning the expander position, its body should be parallel and distant from the palate in 1-2 $\mathrm{mm}$ to avoid contact with the mucosa, and it is necessary to achieve parallelism between the expander long axis and the suture to avoid unleveling of the occlusal plane when the device is activated. Additionally, it should be inserted in a more posterior region, slightly before the limit between hard and soft palate. ${ }^{8,16}$ The mini-implants should be positioned at the region with a greater quantity of the bone available, on the cortical plates of oral and nasal cavities, to favor the primary stability and force dissipation, which should be efficient and uniform. $8,12,16$

Usually, positioning of the expander and mini-implants is planned in the laboratory, ${ }^{8,16}$ with the possibility of errors in the manual accomplishment, owing to stages of transference impression and welding of bands. The difficulty to precisely reproduce the ideal position of MARPE may reduce the clinical effectivity of the device, owing to inflammation of the palatal mucosa and risks of infection of mini-implants. ${ }^{13}$ These situations may be minimized by an efficient and safe digital planning, also reducing the chair time. 

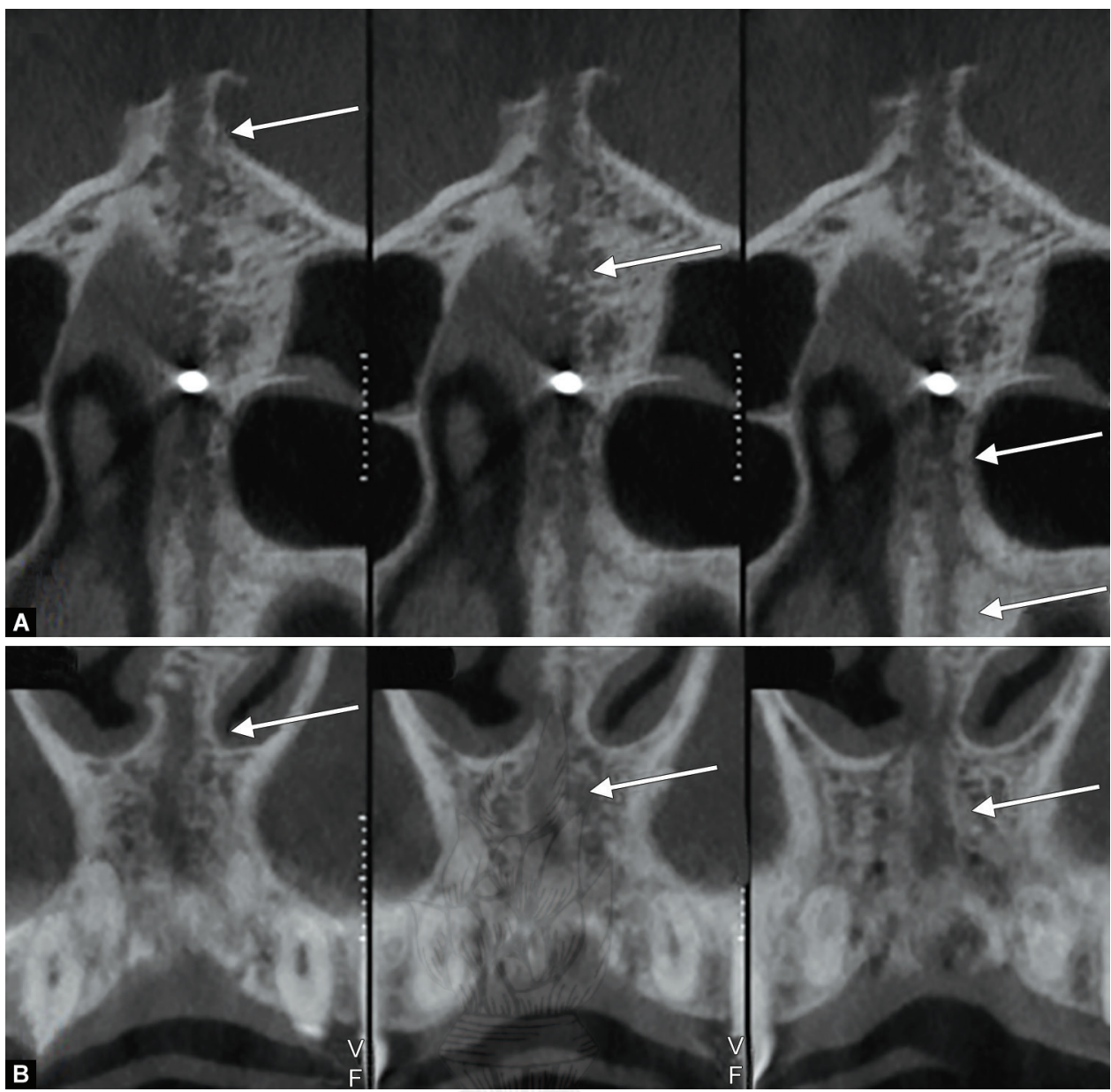

Figs $5 \mathrm{~A}$ and $\mathrm{B}$ : $\mathrm{CBCT}$ after expansion presenting axial (A) and coronal (B) sections, evidencing opening of the midpalatal suture throughout the maxilla extent (white arrows)

It should be mentioned that, in more severe cases, prefabricated expanders present restricted utilization, especially concerning the adjustment in height. This limitation may be compensated by the fabrication of adequate devices by experienced laboratory technicians, ${ }^{18}$ however, the technological advances may overcome this obstacle.

Digital planning in orthodontics using softwares allows simulation of placement of expander and mini-implants, determining the shape, length, diameter, and angulation; it also allows measurement and location of anatomical structures, evaluation of bone density, and visualization of planning on axial, transverse and coronal sections, and also on 3D images.

Utilization of the surgical guide for placement of MARPE is an effective and a new option for treatment with rapid maxillary expansion in patients at the end stage of growth and adults, assuring adequate positioning for placement of mini-implants.

\section{Conclusion and Clinical Significance}

The 3D technological processes assure efficient, accurate, and predictable orthodontic planning, since they standardize the technique and reduce the risks, considering that all processes are digitally prepared, as demonstrated in the present report of fabrication of the MARPE surgical guide, providing better occlusal and respiratory conditions for the patient.

\section{References}

1. Dietrich CA, Ender A, et al. A validation study of reconstructed rapid prototyping models produced by two technologies. Angle Orthod 2017;87(5):782-787. DOI: 10.2319/01091-727.1.

2. Chakraborthy $P$, Krishnamurthy K. Digital Era of Orthodontics: A Review. J Dent Oral Disord Open 2016;2(1):1-3.

3. Ras F, Habets LLMH, et al. Quantification of facial morphology using stereophotogrammetry - demonstration of a new concept. J Dent 1996 Sep;24(5):369-374. DOI: 10.1016/0300-5712(95) 00081-X.

4. Motohashi N, Kuroda T. A 3D computer-aided design system applied to diagnosis and treatment planning in orthodontics and orthognathic surgery. Eur J Orthod 1999;21(3):263-274. DOI: 10.1093/ ejo/21.3.263.

5. Kau CH, Olim S, et al. The Future of Orthodontic Diagnostic Records. Semin Orthod 2011;17(1):39-45.

6. Rheude B, Sadowsky PL, et al. An evaluation of the use of digital study models in orthodontic diagnosis and treatment planning. Angle Orthod 2005;75:300-304.

7. MacGinnis $\mathrm{M}, \mathrm{Chu} \mathrm{H}$, et al. The effects of micro-implant assisted rapid palatal expansion (MARPE) on the nasomaxillary complex - a finite element method (FEM) analysis. Prog Orthod 2014;15:1-15. DOI: 10.1186/s40510-014-0052-y.

8. Brunetto DP, Sant'Anna EF, et al. Non-surgical treatment of transverse deficiency in adults using Microimplant-assisted Rapid Palatal Expansion (MARPE). Dental Press J Orthod 2017;22(1):110-125. DOI: 10.1590/2177-6709.22.1.110-125.sar. 
9. Vandersea BA, Ruvo AT, et al. Maxillary Transverse Deficiency-Surgical Alternatives to Management. Oral Maxillofac Surg Clin North Am 2007;19(3):351-368. DOI: 10.1016/j.coms.2007.04.007.

10. Handelman CS, Wang L, et al. Nonsurgical Rapid Maxillary Expansion in Adults: Report on 47 Cases Using the Haas Expander. Angle Orthod 2000;70(2):129-144.

11. Suri L, Taneja P. Surgically assisted rapid palatal expansion: A literature review. Am J Orthod Dentofac Orthop 2008;133(2):290-302. DOI: 10.1016/j.ajodo.2007.01.021

12. Carlson C, Sung J, et al. Microimplant-assisted rapid palatal expansion appliance to orthopedically correct transverse maxillary deficiency in an adult. Am J Orthod Dentofac Orthop 2016;149(5):716-728. DOI: 10.1016/j.ajodo.2015.04.043.

13. Lee K-J, Park Y-C, et al. Miniscrew-assisted nonsurgical palatal expansion before orthognathic surgery for a patient with severe mandibular prognathism. Am J Orthod Dentofacial Orthop 2010;137(6):830-839. DOI: 10.1016/j.ajodo.2007.10.065.

14. Barriviera M, Duarte WR, et al. A new method to assess and measure palatal masticatory mucosa by cone-beam computerized tomography. J Clin Periodontol 2009;36(7):564-568. DOI: 10.1111/j.1600-051X.2009.01422.x.

15. Barriviera $M$, Minervino $B L$, et al. Tomografia computadorizada volumétrica: um novo protocolo para avaliar a maturação e ossificação da sutura intermaxilar de forma tridimensional e subjetiva. Ortodontia SPO 2017;50(5):424-430.

16. de Oliveira IRM, Guimarães MAC, et al. Marpe - relato de caso e passo a passo da técnica. Ortodontia SPO 2018;51(3):306-313.

17. Moralis A, Zitzmann K, et al. The effects of surgical expansion of the maxillary arch and its consequences for the incisor axis. J Craniomaxillofac Surg 2016;44(5):569-573. DOI: 10.1016/ j.jcms.2016.02.002.
18. Nojima LI, Nojima MDCG, et al. Mini-implant selection protocol applied to MARPE. Dental Press J Orthod 2018;23(5):93-101. DOI: 10.1590/2177-6709.23.5.093-101.sar.

19. D'haese J, Van De Velde T, et al. Accuracy and Complications Using Computer-Designed Stereolithographic Surgical Guides for Oral Rehabilitation by Means of Dental Implants: A Review of the Literature. Clin Implant Dent Relat Res 2012;14(3):321-335. DOI: 10.1111/j.1708-8208.2010.00275.x.

20. Zehnder MS, Connert T, et al. Guided endodontics: accuracy of a novel method for guided access cavity preparation and root canal location. Int Endod J 2016;49(10):966-972. DOI: 10.1111/iej. 12544.

21. Grauer D, Wiechmann D, et al. Computer-aided design/computeraided manufacturing technology in customized orthodontic appliances. J Esthet Restor Dent 2012;24(1):3-9. DOI: 10.1111/j.17088240.2011.00500.x.

22. Chen Y-F, Yoon A, et al. Biomechanical and stress distribution effects of maxillary expansion methods (sarpe, marpe, dome) using finite element model. Sleep Med 2017;1:e57. DOI: 10.1016/ j.sleep.2017.11.159.

23. Wilmes B, Nienkemper $M$, et al. Application and effectiveness of a mini-implant- and tooth-borne rapid palatal expansion device: the hybrid hyrax. World J Orthod 2010;11(4):323-330.

24. Suzuki H, Moon W, et al. Miniscrew-assisted rapid palatal expander ( MARPE): the quest for pure orthopedic movement. Dent Press J Orthod 2016;21(4):17-23. DOI: 10.1590/2177-6709.21.4.017023.oin.

25. Angelieri F, Cevidanes LHS, et al. Midpalatal suture maturation: Classification method for individual assessment before rapid maxillary expansion. Am J Orthod Dentofac Orthop 2013;144(5): 759-769. DOI: 10.1016/j.ajodo.2013.04.022. 